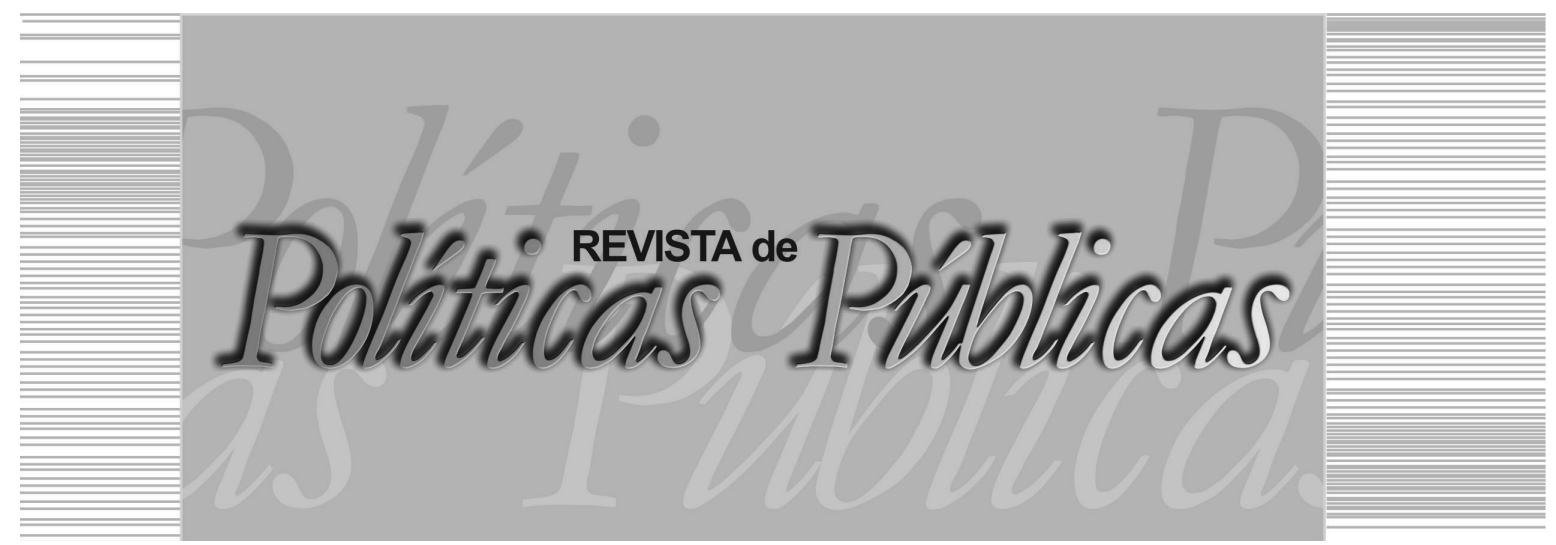

\title{
POLITICAS DE PARTICIPACIÓN CIUDADANA EN ÁMBITOS LOCALES DE ARGENTINA: alcances en términos políticos y de gestión pública
}

\author{
Rodrigo Reynaldo Carmona ${ }^{1}$ \\ Universidad Nacional de General Sarmiento (UNGS) \\ Consejo Nacional de Investigaciones Científicas y Técnicas (CONICET) \\ Barbara Couto ${ }^{2}$ \\ Universidad de Buenos Aires (UBA)
}

\section{Resumen}

El artículo busca examinar los alcances políticos y de gestión pública en la implementación del Presupuesto Participativo y el Programa Argentina Trabaja, en dos municipios de la Provincia de Buenos Aires, San Miguel y San Fernando, en el período 2007-2015. Presenta, así, los principales debates sobre políti-

\footnotetext{
Posdoctor en Ciencias Humanas y Sociales de la Facultad de Filosofía y Letras, Universidad de Buenos Aires (UBA), Doctor en Ciencias Sociales por la Facultad Latinoamericana de Ciencias Sociales, Mención Honorífica (FLACSO-Sede Argentina), Profesor e investigador ordinario del área "Estado, Gobierno y Administración Pública" de Instituto del Conurbano (ICO) de la Universidad Nacional de General Sarmiento (UNGS) e investigador de carrera adjunto del Consejo Nacional de Investigaciones Científicas y Técnicas (CONICET). E-mail: rcarmona@ungs.edu.ar / Universidad Nacional de General Sarmiento - UNGS: Juan María Gutierrez 1150 (entre José León Suarez y Verdi) Conmutador: 4469-7500, Los Polvorines, Pcia de Bs. As. - Argentina C.P. 1613; Consejo Nacional de Investigaciones Científicas y Técnicas - CONICET: Godoy Cruz 2290 (C1425FQB) CABA - República Argentina.

2 Magíster en Política Comparada Latinoamericana de la London School of Economics (Reino Unido), Candidata a doctora en Ciencias Sociales en la UBA, Docente del Área de "Estado y Administración Pública" por la ICO de la UNGS y de Economía Política Argentina por la Facultad de Ciencias Sociales (FSOC) de la UBA. E-mail: bcouto@ungs. edu.ar / Universidad de Buenos Aires - UBA: Marcelo T. de Alvear 2230 - C1122AAJ CABA - Argentina
} 
cas sociales y participativas, gobernanza territorial y trama interjurisdiccional. Luego caracteriza cada municipio seleccionado en términos de las condiciones socioeconómicas del territorio y el escenario político local vis a vis los niveles provincial y nacional de gobierno. Por último, analiza el desarrollo de las políticas seleccionadas en tanto instancias de legitimación del poder político local, como instrumentos de redistribución de recursos al interior del territorio y espacios de gestión pública particular.

Palabras-clave: Políticas sociales y participativas, gestión pública, relación Estado-sociedad, Argentina

\title{
POLICIES OF CITIZEN PARTICIPATION IN LOCAL \\ SCOPES OF ARGENTINA: advances in political and public management terms
}

\begin{abstract}
:
The article seeks to examine the political and public management scope in the implementation of the Participative Budget and the Argentina Works Program, in two municipalities of the Province of Buenos Aires, San Miguel and San Fernando, in the period 2007-2015. It presents, therefore, the main debates on social and participatory policies, territorial governance and interjurisdicional fabric. Next, it characterizes each selected municipality in terms of the socioeconomic conditions of the territory and the local political scenario vis a vis the provincial and national levels of government. Finally, it analyzes the development of selected policies as instances of legitimization of local political power, as instruments of redistribution of resources to the interior of the territory and private public management spaces.

Key words: Social and participatory policies, public management, State-society relationship, Argentina.
\end{abstract}

\section{INTRODUCCIÓN}

Las relaciones Estado-sociedad en Argentina atravesaron importantes cambios durante los últimos 25 años. A la crisis de la matriz estado-céntrica a comienzos de los noventa, siguió, diez años después, la crisis del modelo neoliberal con el estallido social de fines de 2001, alterando los criterios ordenadores de las relaciones sociales y económicas y poniendo al Estado nuevamente en el centro de la escena.

La proliferación de mecanismos de participación ciudadana tiene su comienzo en la era neoliberal en el marco del espacio cedido por el Estado al sector privado y a la sociedad civil. A partir de la crisis de 2001 y en particular desde la Administración nacional de 
Néstor Kirchner, que asume en 2003, la expansión de los instrumentos de participación se inscribe en un proceso de ampliación más general del espectro de políticas públicas en materia social y económica. Es decir, paradójicamente, estos dos movimientos históricos contrapuestos -de repliegue y luego de expansión del Estado- impulsaron aunque de manera diferente, instrumentos de participación social que propiciaron la ampliación de los derechos sociales, mecanismos distributivos a favor de grupos vulnerables y un patrón de ciudadanía más activa en el ámbito local.

En este marco, el artículo da cuenta de los alcances de los procesos de implementación de dos herramientas participativas, el Presupuesto Participativo (en adelante PP) y el Programa Argentina Trabaja (en adelante AT), en dos municipios de la Provincia de Buenos Aires, San Miguel y San Fernando en el período 2007-2015.

El artículo se organiza en cuatro apartados. A continuación se presentan los principales debates sobre políticas sociales y participativas, gobernanza territorial y trama interjurisdiccional. Luego se caracteriza cada municipio seleccionado en términos de las condiciones socioeconómicas del territorio y el escenario político local vis a vis los niveles provincial y nacional de gobierno. A continuación se analiza el desarrollo de las políticas seleccionadas en cada municipio, el Presupuesto Participativo y el Programa Argentina Trabaja abordando los alcances de los procesos de implementación, en tanto instancias de legitimación del poder político local, como instrumentos de redistribución de recursos al interior del territorio, y por último, como vía para la ampliación de derechos ciudadanos. Por último, se reflexiona sobre los desafíos de la implementación de políticas públicas en territorios heterogéneos y de elevados índices de vulnerabilidad social.

\section{DEBATES SOBRE POLÍTICAS SOCIALES Y PARTICIPATIVAS, GOBERNANZA TERRITORIAL Y TRAMA INTERJURISDICCIONAL}

Las reformas económicas de orientación neoliberal desplegadas en América Latina en los noventa, y el proceso de descentralización y transferencia de funciones del nivel nacional hacia los niveles subnacionales fueron plasmando nuevas dinámicas en el plano territorial. Estas políticas tuvieron un impacto de carácter doble sobre los gobiernos locales. Por un lado, los municipios han sido 
testigos de una ampliación en sus funciones y responsabilidades y en menor medida en sus atribuciones, debiendo proveer de este modo un conjunto mayor y más heterogéneo de servicios a la comunidad, sin que ello haya implicado un incremento en sus recursos técnicos, jurídicos, económicos, humanos y administrativos acorde al crecimiento en la cantidad y complejidad de sus tareas. Por otro, se ha resignificado y en alguna medida jerarquizado el ámbito local. Los municipios han comenzado a tener una mayor importancia en razón de la cercanía dada entre gobernantes y gobernados, posibilitando de este modo el nacimiento de nuevas prácticas y formas organizativas de resolución de problemas y conflictos como estrategias para sobrellevar la retirada del Estado, y sobre todo del Estado Nacional. (CRAVACUORE; ILARI; VILLAR, 2004; CABRERO MENDOZA, 2005; CARMONA, 2012a, 2012b).

Tal situación dio lugar a la aparición de un conjunto de nuevos mecanismos de política y gestión públicas más participativas, en un escenario de cuestionamientos a las formas de representatividad tradicionales. Precisamente, el Presupuesto Participativo o el Programa Argentina Trabaja, aparecen como herramientas innovadoras que buscan replantear el modo de planificar y gestionar el territorio. El PP lo hace incorporando las perspectivas de distintos actores en un escenario de creciente complejidad y promoviendo formas más directas y vinculantes en los asuntos de gobierno. (SÁNCHEZ, 2002; CABANNES, 2005).

Por su parte, el AT en tanto programa de generación de empleo, involucra a las organizaciones territoriales en diferentes aspectos de la gestión del Programa en cooperación con el municipio, por ejemplo operando como mediadores en la elección de los grupos de destinatarios.

Así, frente a políticas de corte más generalista en el tratamiento de las problemáticas locales se subraya la importancia de considerar las especificidades territoriales a fin de adaptar las agendas y modalidades de intervención a las particularidades de cada lugar. Esto permite propiciar procesos de cambio sobre la base de un enfoque estratégico que combine una lógica de funcionamiento transversal e intersectorial y el desarrollo de diversas interacciones/ redes entre los actores locales desde una perspectiva de gobernanza. (BLANCO; SUBIRATS, 2008). 


\section{POLÍTICAS DE PARTICIPACIÓN CIUDADANA EN AMBITOS LOCALES DE}

ARGENTINA: alcances en términos políticos y de gestión pública

Puede aseverarse entonces que, en líneas generales, la gobernanza en la literatura especializada plantea el pasaje gradual de una modalidad de actuación gubernamental sostenida en la primacía excluyente del gobernante en términos de la dirección y conducción política, a un escenario donde se establecen mayores grados de interacción y dependencias mutuas entre las instancias político-institucionales y sociales (BLANCO; GOMÁ, 2006; AGUILAR VILLANUEVA, 2007; CARMONA, 2012a; MARTÍNEZ; ARENA, 2013). De este modo, el sentido básico que identificamos a partir del concepto es la decisión política, más o menos voluntaria, por parte de las autoridades de generar una apertura en las decisiones de gobierno y renovar/democratizar el vínculo Estado-sociedad, incorporando a los actores no estatales (sea esto por su representatividad o importancia en relación al conjunto de intereses sociales implicados) en el despliegue de políticas públicas.

La creciente democratización de la vida pública y los procesos estructurales plasmados en las últimas décadas implican por lo menos dos procesos: i) el reconocimiento de la necesidad de descentralizar la gestión de los asuntos públicos en instancias más cercanas a la ciudadanía; ii) y derivado de lo anterior, una mayor participación de los diversos actores no gubernamentales en el proceso gubernamental, desde el diseño hasta la ejecución y evaluación de las políticas públicas (JORDANA, 2003; LOZANO GONZÁLEZ, 2009; CANTO CHAC, 2008).

Los ejes para el análisis de este debate pueden rastrearse así en las transformaciones atravesadas en las últimas décadas por el Estado y la sociedad, que plantea nuevas necesidades en términos de coordinación de los actores. Lechner (1997) reconoce así dos paradigmas tradicionales de coordinación: 1) la coordinación política desarrollada por el Estado y establecida como centralizada, pública, jerárquica y deliberada; 2) la coordinación generada por mecanismo de mercado, que tiende a acciones de tipo descentralizadas, privadas, horizontales y no deliberadas - sobre la base de un equilibrio espontáneo de los intereses. El predominio casi absoluto que la esfera estatal detentaba en el plano político y social hasta hace algunas décadas se ve cuestionado entonces por la envergadura y escala de los problemas. Por un lado, el funcionamiento burocrático y saber técnico tradicional en un escenario de mayor complejidad y restricciones encuentra límites para desarrollarse y requiere de la complementación de otros saberes (sociales, culturales) e interacción con diversos 
actores sociales para generar mejorares políticas. El funcionamiento electoral, como único mecanismo legitimador del accionar del Estado, aparece también puesto en cuestión y obliga a pensar en formas más participativas de construcción de consenso y poder político. Al mismo tiempo, las modalidades que asume la acción colectiva se diversifican y los partidos políticos y otras expresiones de intereses generales tradicionales pierden fuerza como instancias aglutinadoras. Ello determina que la crisis del Estado como centro exclusivo de representación, planificación y conducción de la acción pública, como las debilidades propias del mercado para establecer un orden social equilibrado, abran la discusión respecto a la relevancia de modalidades de coordinación que se adecuen a la complejidad social existente y den pie a nuevos esquemas de participación ciudadana.

Es importante aclarar, no obstante, que la participación ciudadana adquiere formas distintas y presenta características particulares según los fines, actores y contextos en los cuales se lleva a la práctica.

En esta línea, Cunill Grau (1991) resalta que las modalidades que asuma la participación ciudadana dependerán de dos factores: 1) los niveles y ámbitos en los que recaiga la participación; 2) el carácter de la intervención de los ciudadanos en las actividades y/o órganos públicos. De este modo, la participación ciudadana puede darse en un plano más propiamente político como de gestión. El plano político es el que se relaciona con el despliegue de opciones o decisiones de carácter público, esto es, las actividades de gobierno. En este plano pueden apreciarse niveles de participación más agregados o macro (decisiones que involucran al conjunto de la comunidad y al régimen político), intermedios (políticas de alcance sectorial o regional) y micro (decisiones vinculadas con el accionar cotidiano de individuos y grupos pequeños). El plano de la gestión es el que se vincula más puntualmente con el desarrollo de bienes y servicios públicos y con las actividades de apoyo a éstos. Este tipo de participación puede plantearse de diferentes formas: participación consultiva y/o asesora (opinión o declaración de conocimiento que no obliga al sujeto que adopta la decisión), participación resolutiva y fiscalizadora (intervención en el curso de la actividad pública y carácter obligante para la administración) y participación en la ejecución (se toma parte en forma directa en la realización de una actividad y/o prestación de un servicio). 


\section{POLÍTICAS DE PARTICIPACIÓN CIUDADANA EN AMBITOS LOCALES DE}

ARGENTINA: alcances en términos politicos y de gestión pública

El carácter que la participación ciudadana adquiere respecto a la toma de decisiones públicas puede plantearse así en términos de: información (los actores son informados de las acciones que se realizarán), consulta (los actores son consultados respecto a las acciones a desarrollarse y pueden aprobar o no lo proyectado) y cogestión/ codecisión (los actores son parte en la elaboración de las acciones y la toma de decisiones).

Finalmente, el origen del impulso hacia la participación constituye también un aspecto determinante dado que es posible diferenciar entre dos tipos: la acción ciudadana, que comienza y es controlada por los propios actores sociales en relación a los objetivos y fines que ellos determinan; la implicación ciudadana, cuyo inicio y control es desplegado por el Estado con el fin de conseguir apoyo y mejorar las decisiones, programas o servicios.

Los motivos que explican en los últimos tiempos la proliferación de experiencias participativas - más tradicionales o innovadoras- se relacionan básicamente con las restricciones que presentan las formas clásicas de participación previstas en los modelos de democracia representativa.

La democracia representativa, tal como sugiere Annunziata (2011) pareciera entonces no poder por sí misma sostener su legitimidad, lo cual aparece como punto de acuerdo de una gran variedad de estudios académicos que se han referido a la crisis de la representación. La Ciencia Política desde hace varios años ha tomado este núcleo problemático de divorcio entre la ciudadanía y sus representantes. Un clásico trabajo en este sentido es el de Manin (1998), para el que la personalización de la política, la fluctuación del voto, la creciente importancia dela imagen de los candidatos en los medios de comunicación no eran síntomas de una crisis de la representación misma, sino de un formato específico del gobierno representativo, una metamorfosis de la representación.

Tanto los programas sociales como los participativos plantean en este punto la cuestión de la autonomía relativa de los actores de la sociedad civil vis a vis el Estado. Las asimetrías de poder recursos económicos, políticos, institucionales- son muy marcadas, tanto más cuanto mayor es el nivel de vulnerabilidad social de los grupos sociales involucrados. 
Mientras que algunos trabajos proponen la dicotomía autonomía-cooptación como los trabajos que abordan los problemas de clientelismo político derivados de la implementación de políticas sociales, otros señalan que dicha dicotomía no expresa la complejidad de la relación política en cuestión. En cambio, pensar en un continuo entre autonomía-heteronomía permite reflexionar sobre la tensión entre las agendas de los actores sociales y las agendas gubernamentales, tanto locales como de otros niveles de gobierno. (SVAMPA, 2009; LOZANO, 2012; NATALUCCI, 2010; FORNI; CASTRONUOVO, 2014).

Por otra parte, en las últimas dos décadas en países federales como la Argentina, la gestión Nación-Provincias-Municipios ha transformado su injerencia,

\section{[...] pasando de un modelo que suele denominarse como dual - en donde el ámbito nacional por un lado, y las provincias y munici- pios por otro, actuaban de forma independiente - a otra conocida como federalismo cooperativo o coordinado, en el cual los tres ni- veles de Estado trabajan de manera conjunta (CAO, 2007, p. 17).}

Esta transformación ha propiciado la cogestión entre niveles de gobierno que en ocasiones se superponen con procedimientos complejos en especial en el proceso de toma de múltiples decisiones en la medida que se amplían los actores que intervienen no solo al interior del Estado sino también de la sociedad civil en las diferentes etapas de la implementación. Si bien como señalamos el proceso se vuelve complejo y abierto a diferentes puntos de veto, la intervención multiactoral fortalece la acción estatal en términos de legitimidad política y social.

\section{CARACTERIZACIÓN SOCIOECONÓMICA DEL TERRITORIO}

La Provincia de Buenos Aires constituye un territorio sumamente complejo: concentra el $38 \%$ de la población del país y el $37 \%$ del padrón electoral nacional, representa el $32 \%$ del valor de producción del país y el $45 \%$ de la industria manufacturera. Posee autonomía financiera dado que sus recursos propios representan el $40 \%$ de sus ingresos totales. Por otro lado, es la segunda provincia más densamente poblada, su territorio es sumamente heterogéneo en términos socioeconómicos - desigual capacidad productiva y condiciones de vida de la población-, en particular entre las zonas más ur- 
banizadas, los 24 partidos que constituyen el denominado Conurbano Bonaerense (que concentra el mayor índice de pobreza del país) y las zonas rurales, lo que matiza su situación de provincia próspera. Los dos municipios seleccionados pertenecen al Conurbano Bonaerense y se ubican en la segunda corona del área metropolitana, con una población de más de 280 mil habitantes en San Miguel y más de 163 mil habitantes en San Fernando.

En términos sociales, una década de crecimiento sostenido ha permitido a la Provincia reducir la proporción de hogares con Necesidades Básicas Insatisfechas (NBI), de 13\% en 2001 a 8\% en 2010. Los datos de NBI para el Conurbano Bonaerense se reducen de $14.5 \%$ en 2001 a 9.4\% en 2010, ubicándose los casos seleccionados por debajo de ese valor (San Miguel con 8,2\% y San Fernando con 8,6\%). Como reflejo de la heterogeneidad del territorio, los indicadores de hogares que en el último Censo Nacional 2010, carecen de un sistema público de desagüe muestran un déficit moderado en San Fernando (22\%) y elevadísimo en San Miguel (66\%) en comparación al de la Provincia (53\%) y al correspondiente al nivel nacional (48\%) (Tabla 1).

Tabla 1 - Indicadores sociales

\begin{tabular}{c|c|c|c}
\hline Municipio & $\begin{array}{c}\text { Nec. Básicas } \\
\text { Instat. }\end{array}$ & $\begin{array}{c}\text { Hogares sin red } \\
\text { pública cloacal }\end{array}$ & $\begin{array}{c}\text { Tasa de } \\
\text { Desempleo }\end{array}$ \\
\hline SAN FERNANDO & 8.6 & 22,33 & 6.1 \\
\hline SAN MIGUEL & 8.2 & 65,91 & 6.0 \\
\hline
\end{tabular}

Fuente: Elaboración propia, en base a datos del INDEC. Censo Nacional de Población, Hogares y Viviendas 2010.

Si bien los indicadores de NBI y desempleo denotan similitudes entre ambos partidos, el déficit de infraestructura cloacal evidencia una brecha significativa entre ambos.

San Miguel posee un desarrollo productivo bastante periférico, con un desempleo del $6.1 \%$. En su estructura económica, los servicios tienen un peso mayor $(85,6 \%)$ que la producción de bienes, predominando el comercio, el transporte y los servicios inmobiliarios; sin tener el partido un peso significativo dentro del mapa sectorial de la Provincia en ningún sector de actividad en particular.

En cuanto a la heterogeneidad social del territorio, diversos autores señalan que San Miguel se destaca por la existencia de 
islas con alto nivel económico en un entorno de gran vulnerabilidad social. En la década del noventa experimentó un aumento poblacional elevado - del 19\%- siguiendo un patrón de segregación socioeconómica, con una fuerte concentración espacial de sectores vulnerables, en particular sectores de débil inserción en el mercado de trabajo. (SUAREZ; GROISMAN, 2007). Presenta una estructura habitacional que sigue este mismo patrón, con 31 barrios cerrados que ocupan una superficie de 1.054 ha, y en el otro extremo de la segmentación urbana, 11 asentamientos ilegales que cubren una superficie de 258 ha, entre los que se encuentra la villa de emergencia más grande del Conurbano. (SUAREZ; GROISMAN, 2007).

San Fernando, posee una estructura productiva más especializada que San Miguel, con un desempleo del 6.1\%. El territorio está formado por dos áreas netamente diferenciadas: una sección continental de $23 \mathrm{~km}^{2}$, densamente poblada y una sección de islas del Delta del Paraná de más de $900 \mathrm{~km}^{2}$ (cifra aproximada sujeta a variación por el avance deltaico). La producción de bienes tiene un peso más importante $(60.8 \%)$ que la de servicios, con mayor incidencia de la producción industrial (2.3\%) en el total de la Provincia, similar al peso de otros partidos relevantes como Quilmes o Bahía Blanca.

En la zona continental, se ha desarrollado una trama industrial importante vinculada, por un lado, a la actividad naval y náutica y, por el otro, a la industria maderera, las cuales confieren fuerte identidad al territorio. Dentro de estos sectores, predominan las empresas pequeñas y medianas: los astilleros y talleres navales, por citar algunos ejemplos de la actividad náutica; y los talleres y fábricas de procesamiento de la madera en el otro caso, que concentra el 14\% del empleo y el $30 \%$ de las empresas industriales. Las actividades más concentradas - en pocas grandes empresas - son la alimentación y la producción ligada al neumático.

San Fernando posee 17 barrios carenciados dentro del área continental y no se cuenta con información sobre las islas, cuya población vive generalmente en condiciones precarias, en tanto carece de servicios básicos y está débilmente integrada al entorno productivo continental. 
POLÍTICAS DE PARTICIPACIÓN CIUDADANA EN AMBITOS LOCALES DE

ARGENTINA: alcances en términos politicos y de gestión pública

\section{LAS POLÍTICAS IMPLEMENTADAS}

\subsection{El Presupuesto Participativo}

El programa empieza a desarrollarse en Argentina luego de los estallidos sociales de fines del año 2001, en un escenario marcado por una grave crisis de representación política y social y en un estado de colapso económico. A partir de las primeras experiencias, que datan de 2002, los gobiernos locales argentinos han, paulatinamente, desarrollado el Presupuesto Participativo, con un salto cuantitativo muy importante en la cantidad de experiencias a partir de 2008 , año en que se pasa de diez a veintiún municipios con PP y creciendo posteriormente de manera gradual y sostenida en el tiempo.

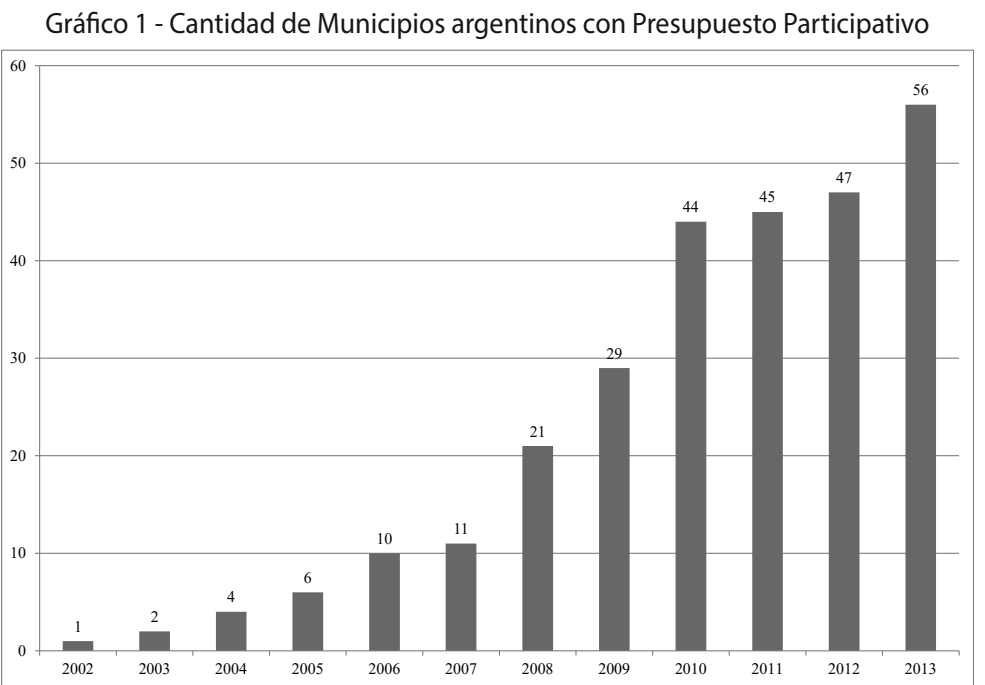

Fuente: Elaboración propia, a partir de datos de la Red Argentina de Presupuesto Participativo.

A toda esa expansión han contribuido, en parte, también el involucramiento y el asesoramiento técnico en la promoción del PP de la Administración nacional anterior (2003-3015) a través de un programa articulado entre la Secretaría de Relaciones Parlamentarias de la Jefatura de Gabinete de Ministros y de la Secretaría de Asuntos Municipales del Ministerio del Interior y Transporte, denominado Programa Nacional de Presupuesto Participativo, que entre otras acciones en la materia han creado la Red Argentina de Presupues- 
to Participativo (RAPP) como foro de intercambio de experiencias entre los distintos municipios que desarrollan o están interesados en desarrollar esta política. A su vez, colaborando con ese programa participan organizaciones de la sociedad civil, organismos y expertos internacionales y algunas universidades públicas entre las que se encuentra la Universidad Nacional de General Sarmiento (UNGS).

En la Región Metropolitana de Buenos Aires puntualmente, como se observa en el cuadro del anexo, en 2010, diez municipios implementan el PP, experiencias que emergen en su mayoría después de 2008.

En San Fernando específicamente se implementó entre 2008 y 2011 a propuesta del Ejecutivo, regulado por Decreto municipal. Se buscó generar una herramienta que permitiera ampliar los canales de consulta vecinal, profundizando la democracia participativa e incorporando al vecino a la gestión pública.

Los proyectos en su mayoría consisten en obras de infraestructura, ya sea en espacios públicos o semipúblicos (reacondicionamiento de entidades intermedias, escuelas, jardines de infantes) por lo que, el monto de las intervenciones, es ciclo tras ciclo, más elevado. Los niveles de participación son elevados. 3900 vecinos participaron de la Feria de Proyectos donde se presentan las propuestas formuladas por la ciudadanía previamente a la votación. San Fernando tiene a su vez, un PP joven que es coordinado por el mismo equipo. Aunque con dificultades para sostenerlo en el tiempo y con algunas complicaciones administrativas, en el año 2010 participaron 1800 jóvenes proponiendo y votando diversos proyectos. El PP Joven se entiende como continuidad del Parlamento joven, un mecanismo utilizado por el Municipio durante 10 años.

La participación va creciendo durante los cuatro ciclos que perdura la experiencia y va madurando la visión de la ciudadanía en relación al diseño de proyectos. En la segunda edición el municipio introduce algunos cambios para impedir que algunas organizaciones acaparen el proceso limitando la participación de vecinos independientes, poniendo topes del $50 \%$ en los montos de los proyectos y limitando la presentación de un mismo proyecto dos años seguidos, por ejemplo para la ampliación de infraestructura de una organización vecinal. 


\section{POLÍTICAS DE PARTICIPACIÓN CIUDADANA EN AMBITOS LOCALES DE}

ARGENTINA: alcances en términos políticos y de gestión pública

Los montos destinados son elevados, en comparación con muchos municipios de la región. En 2010 se destina un 2\% del presupuesto municipal, que asciende a U\$S 500.000, para la implementación de 11 proyectos mayoritariamente vinculados al mejoramiento de la infraestructura urbana. En cuanto al impacto redistributivo del PP, en el caso de San Fernando no se incorporó en el diseño de la herramienta ningún mecanismo que compensara a zonas desfavorecidas, aunque varias organizaciones de la región más pobre han logrado movilizar proyectos para dictar capacitaciones en oficios en zonas con elevados índices de desempleo, por ejemplo.

En San Miguel, la experiencia del PP se constituye como caso paradigmático. La iniciativa comenzó a desarrollarse como el corolario de un conjunto de factores que fueron convergiendo desde el año 2005 en adelante: 1) la debilidad institucional de un gobierno municipal desprestigiado y con mala imagen (Oscar Zilocchi, intendente desde 2003); 2) un recambio interno dentro del Partido Justicialista local, que se expresaría paulatinamente en el Concejo Deliberante, y con la asunción de un nuevo intendente que apoyaría esta herramienta para ganar legitimidad en su gestión (Joaquín De la Torre, en 2007), y 3) la presencia de universidades, organizaciones sociales y movimientos políticos ${ }^{1}$, con trabajo territorial y de resistencia a escala nacional-local, y cierta capacidad de movilización y veto (CARMONA, 2012b).

Con la asunción del nuevo gobierno local de Joaquín De la Torre, y luego de un trabajo sostenido de los distintos actores implicados, la reglamentación se terminó aprobando por decreto a principios de 2008. El proceso induce en el municipio un proceso de desconcentración en zonas que constituyen un insumo esencial como primer paso hacia la planificación territorial. En total, participarían del proceso del PP más de dos mil vecinos, y con una ejecución de gran parte de los primeros proyectos. Por otro lado, el porcentaje afectado al PP pasaría al $7 \%$ de los recursos municipales (el porcentaje más elevado de todos los casos de la Región) y se establecería una pauta de redistribución del presupuesto, según necesidades básicas insatisfechas. La mitad del presupuesto se distribuye por igual entre los foros territoriales y la mitad se distribuye entre los foros que presentan mayores índices de NBI.

La dinámica de participación fue variando con el correr de los ciclos. Hacia 2012 se buscó legitimar la herramienta ampliando 
considerablemente la cantidad de vecinos participantes. Este proceso llevó a expandir la participación de baja intensidad (más vecinos participan a través de la votación) descuidando las dinámicas de alta intensidad (en asambleas) donde se abre el debate a otras temáticas.

Otro rasgo sobresaliente de San Miguel es la promoción de temáticas novedosas de abajo hacia arriba, que rompen con la agenda del municipio generando iniciativas innovadoras. Los proyectos de apoyo escolar y violencia de género propuestos por el foro Don Alfonso, ubicado en una de las zonas más desfavorecidas del municipio, constituyen casos relevantes en este sentido. Estas propuestas exigen arreglos institucionales diferentes a los que habitualmente rigen en las propuestas de PP (iluminaria, infraestructura urbana, deporte, etc.), dado que constituyen temáticas interdisciplinarias que involucran a diferentes áreas del municipio. Más importante aún, el abordaje de estas propuestas al interior de los gabinetes impulsa necesariamente discusiones que no se habían dado en estos espacios, abriendo la posibilidad de incorporar los temas en la agenda general del municipio, como lo ilustra la conformación de la mesa intersectorial de violencia familiar en el Municipio.

Como ilustra el caso de San Miguel, el PP puede - aunque solo en algunos casos lo logra - habilitar procedimientos innovadores, como por ejemplo la aplicación criterios redistributivos a favor de grupos vulnerables o la movilización de nuevos derechos en temáticas de género o violencia familiar que exijan un abordaje integral por parte del municipio.

En síntesis, en ambos casos la herramienta opera predominantemente como un instrumento de legitimación de las autoridades municipales, apoyándose en el factor de proximidad para fortalecer los vínculos entre gobernantes y gobernados. El estudio de múltiples experiencias de PP (LÓPEZ ACCOTTO et al., 2014; CARMONA; COUTO 2015) muestra que generalmente son limitadas las temáticas que pueden convertirse en proyectos, por la exigencia de ejecución presupuestaria anual, por la tradicional compartimentación de las áreas municipales que limita los abordajes integrales que requieren algunas temáticas (niñez, género, etc.) y finalmente por la resistencia de las gestiones municipales a abordar temáticas novedosas que escapen a los temas tradicionales de su agenda. 
POLÍTICAS DE PARTICIPACIÓN CIUDADANA EN AMBITOS LOCALES DE

ARGENTINA: alcances en términos politicos y de gestión pública

\subsection{EI Programa Ingreso Social con Trabajo PRIST-Argentina Trabaja}

En el año 2009, a pesar del crecimiento económico sostenido desde 2002, persisten altos índices de desocupación y de pobreza por lo que surgen desde el Gobierno nacional dos programas sociales que se orientarían a paliar esta situación, la Asignación Universal por Hijo para la Protección Social (AUH) y el Programa Ingreso Social con Trabajo Argentina Trabaja (PRIST-AT), que fueron mencionados anteriormente. EL AT apuntaba a promover la inclusión social a partir del desarrollo local sostenible fortaleciendo el asociacionismo y la economía social.

Según un informe del Ministerio de Desarrollo de la Nación, en 2011 se incorporaron al programa 189.000 personas al Programa, conformando 2022 cooperativas que habilitaban a sus beneficiaros a acceder al sistema de salud a partir de un aporte que realizaba con el aporte denominado Monotributo social, que contemplaba el mismo programa.

En cuanto a la ejecución del Programa, el Ministerio de Desarrollo de la Nación transfiere fondos a los Entes Ejecutores (Provincias, Municipios, Cooperativas y/o Asociaciones mutuales) para la adquisición de herramientas y materiales que representan el $30 \%$ de los fondos totales destinados a cada obra. El 70\% restante corresponde al subsidio que reciben los receptores en concepto de excedentes, que se transfiere directamente a los miembros de las cooperativas a través de una tarjeta bancaria.

El monto ejecutado a nivel provincial entre 2009-2011 ascendía a $\$ 420.039$ pesos (U\$S98.139) y el ejecutado a nivel municipal, alcanzaba los 2.266.434 pesos (U\$S528.306) $)^{2}$.

En la Provincia de Buenos Aires, de un total de 43 municipios, 30 implementaron el AT (ARGENTINA, 2011), entre los que se encuentran San Fernando y San Miguel.

En el caso de San Fernando, al comienzo de la implementación del Programa se hizo una convocatoria abierta, incluso a las organizaciones políticas de diverso signo partidario. Se realizaron tres operativos masivos de registro e inscripción. Se sistematizó entonces una base de datos de cooperativas que habían funcionado en el territorio con diversos planes sociales (como Plan Más Vida, planes alimentarios, etc). 
Una vez analizada la factibilidad y alcance de los recursos que Nación destinaría, se estableció un cupo de aproximadamente 1400 personas en el programa Argentina Trabaja. Una vez inscriptos los cooperativistas y organizados de acuerdo al Programa, se trabajó en la capacitación de esas personas en relación a cuestiones de seguridad en el trabajo y en cuestiones que tienen que ver con cooperativismo. Luego se conformaron los consejos de administración en base a las experiencias que ya existían desde las cooperativas de viviendas.

La base de cooperativas más chicas de 16 integrantes, que ya estaban conformadas con su Consejo de Administración fue ampliada a cooperativas de 60 integrantes. Con 22 cooperativas conformadas se logró llegar al número total de 1400 cooperativistas en San Fernando.

Las cooperativas realizan obras de mediana y baja complejidad en los barrios del distritito, en tareas diversas de saneamiento, infraestructura urbana, mejoramiento de espacios verdes, infraestructura comunitaria, mejoramiento habitacional en viviendas particulares y también del hábitat.

Si bien existían cooperativas desde principios de la década, con el Argentina Trabaja se multiplicó la cantidad de participantes y la mayoría de las cooperativas quedaron funcionando bajo dicho Programa.

Con el cambio de gestión en 2011 y la asunción de un gobierno local de signo político contrario, el municipio fue desplazado como interlocutor en la ejecución del programa, articulando la Nación con otros espacios del territorio donde no tuviera que articular con el municipio. En este sentido, avanzan sobre espacios aledaños al ferrocarril Mitre en San Fernando, que tiene jurisdicción nacional, trabajan en clubes y escuelas que dependen de la Provincia de Buenos Aires, para lo que establecieron un convenio con la Dirección General de Escuelas. En esta nueva etapa el número de cooperativas asciende a 48 y tiene la particularidad que los trabajos que realizan no son exclusivamente en el territorio de San Fernando, sino que muchas veces los cooperativistas son trasladados fuera del Partido.

De modo que San Fernando experimenta dos etapas en la implementación del Programa. En la primera, el municipio constituye la vía de ingreso de la Nación al territorio mientras que en la segun- 
da, con la asunción de un nuevo gobierno de signo político opositor, la Administración nacional establece un puente para evitar articular con el gobierno local, estableciendo convenios directamente con otros Entes Ejecutores locales, ampliando en esta etapa el número de cooperativas.

En el caso de San Miguel, pueden identificarse tres etapas en la implementación del Programa. En una primera etapa se organizaron dos líneas de implementación a través de Entes Ejecutores diferentes. Una, que involucra al Municipio y otra que involucra a la Provincia. En cada caso, las organizaciones que integran las cooperativas son diferentes.

En la primera etapa del Programa en San Miguel, el municipio manejó de 2009 a 2011 el $60 \%$ de las cooperativas del programa y la Provincia el $40 \%$. El gobierno provincial y el gobierno municipal bajan al municipio distintos módulos de cooperativas. Esta modalidad fue rediseñada luego de 2011. De acuerdo a la entrevista realizada a Germán Quinteros, coordinador territorial del Municipio de San Miguel, la parte del programa que coordinaba el gobierno provincial en el municipio fracasó porque esa organización no estuvo en el territorio, no llegaban las herramientas ni los recursos en tiempo y forma. Por tal motivo, existieron ciertos desencuentros con las organizaciones sociales y políticas que conformaron las cooperativas que funcionaban bajo la línea de la Provincia.

En esta primera etapa de la implementación del programa en San Miguel, hubo problemas en el armado de las cooperativas. Las personas se presentaban y anotaban para formar parte del programa, y el municipio armó las cooperativas sin tener en cuenta el domicilio de las personas. De esa manera, las cooperativas quedaron conformadas por personas de distintos barrios y eso dificultó el funcionamiento real de las cooperativas en los barrios.

Es por eso que en el transcurso de la implementación del programa se fueron armando cooperativas ad hoc (sin contar con personería jurídica ni estar inscriptas como tales en el programa) según el territorio al que pertenecían los beneficiarios y desde el municipio se organizó el trabajo desde los coordinadores territoriales en las 26 zonas en las que se dividió el municipio, que permitieron desde 2012, organizar el Programa en 70 cooperativas de aproximadamente 30 beneficiarios cada una. 
La tercer etapa comienza luego de las elecciones intermedias de 2013, a partir de las cuales el Intendente de San Miguel se vuelve opositor al Gobierno Nacional al establecer alianza con el Frente Renovador, nueva fuerza política que logra aglutinar buena parte de los Intendentes conurbanenses. La Nación deja de articular con el Municipio y se apoya en otras organizaciones sociales y entidades universitarias de la zona para desarrollar el AT.

En síntesis, existe en ambos casos una fuerte imbricación de la política nacional en la implementación del programa que puede fortalecer al Municipio en su articulación con las organizaciones del territorio pero en un vínculo de extrema dependencia respecto del nivel Nacional que está supeditado al apoyo político, lo que se evidencia en el análisis de ambos casos.

Por otro lado, existe otra tensión que es interna al Programa, que se presenta entre los objetivos planteados por las políticas sociales y participativas que buscan reforzar desde el nivel estatal la organización y autonomía del tejido asociativo al mismo tiempo que generan una dependencia del financiamiento público estatal. (ARCIDIÁCONO; KALPSCHTREJ; BERMÚDEZ, 2014; GUIMÉNEZ; HOPP, 2012). La autonomía de las organizaciones que constituyen la cooperativa es muy limitada en tanto por un lado la definición de los reglamentos internos por ejemplo está fuertemente condicionada por el Programa, y por otro lado las cooperativas no se constituyen espontáneamente en torno a objetivos propios y previos de las organizaciones, sino en torno a los objetivos que plantea el AT, quedando atadas al destino del mismo.

\section{CONCLUSIÓN}

Hemos señalado al comienzo que ambas políticas funcionan como instancias de legitimación del poder político local. En el caso del PP, como instrumento de acercamiento a la ciudadanía y de apertura de debates más o menos abiertos según el caso. En el Programa AT, el proceso de legitimación se consolida cuando los municipios logran el control del proceso de implementación y de asignación de los recursos al interior del territorio. Lo contrario sucede cuando la Nación saltea al gobierno local acumulando poder sobre el territorio sin la venia del municipio e incluso compitiendo con él.

El alcance en términos de redistribución de recursos es significativo. En el caso del AT, como señalamos los montos destinados 
a los municipios de la Provincia de Buenos Aires son más que cuantiosos. En el caso del PP los municipios destinaban proporciones importantes del presupuesto al debate popular. En términos de la competencia dentro del territorio por los recursos que asigna el Estado, la puja suele darse en el PP, entre asociaciones y vecinos independientes o entre zonas que buscan acaparar los recursos destinados en el Programa. Sin embargo, los diseños regulatorios van estableciendo pautas en este sentido que limitan la puja por los recursos. Lo más significativo aparece cuando los diseños de PP contemplan mecanismos con sesgo distributivo a favor de grupos vulnerables, como en el caso de San Miguel.

En ambos casos analizados, ambos programas promueven procesos de gobernanza territorial al fortalecer a las organizaciones sociales y políticas del territorio en la relación con el Estado. En relación al PP, la tensión se plantea más entre la agenda de los actores sociales y la agenda municipal. Allí se puede vislumbrar una tendencia bastante generalizada a limitar el abanico de temáticas que pueden someterse al debate público, limitando los márgenes de participación social y encorsetando las discusiones a las habituales temáticas de la agenda tradicional municipal. Hemos señalado que las asimetrías en el control de los recursos con el gobierno son significativas. Tanto el gobierno local como el nacional o el provincial mantienen un control sobre los procesos de implementación fortaleciendo la capacidad organizativa de la sociedad civil pero generando vínculos de dependencia importantes, en especial en el caso de Argentina Trabaja donde se pueden habilitar escenarios de cooptación, desarrollando una infraestructura institucional funcional a los fines electorales.

Señalamos también que la heterogeneidad económico-social del territorio del Conurbano condiciona los procesos de implementación de las políticas sociales y participativas. La autonomía financiera de los municipios, que está fuertemente correlacionada con su potencial económico, condiciona las relaciones interjurisdiccionales presentando mayores márgenes de acción a aquellos municipios que dependen menos de las transferencias de los niveles superiores de gobierno.

Finalmente, las organizaciones sociales y los referentes territoriales del nivel municipal, han ido adquiriendo una centralidad importante en las políticas sociales a través de su activa participa- 
ción como mediadores e instrumentadores de las políticas, en tanto adaptadores de los dispositivos institucionales de niveles superiores de gobierno, a la escala local. Estos actores de escala territorial funcionan como un vehículo de aproximación política a los territorios populares, desde el nivel municipal o desde el nivel nacional cuando éste interviene a través de una oficina del Ministerio o desde una organización alineada con la fuerza política gobernante en el nivel nacional, tanto en connivencia con el nivel municipal o a veces puenteándolo en los casos de no-alineamiento político. Hemos debatido así cómo en el doble proceso de fortalecer-controlar el territorio, la gestión multinivel y la impronta de gobernanza territorial que poseen las nuevas políticas sociales y participativas, emergen viejos y nuevos desafíos en el camino de transformación de realidades sociales complejas y desiguales dentro del territorio del Conurbano Bonaerense de la Provincia de Buenos Aires.

\section{REFERENCIAS}

AGUILAR VILLANUEVA, L. El aporte de la política pública y la Nueva Gestión Pública a la gobernanza. In: CONGRESO INTERNACIONAL DEL CLAD SOBRE LA REFORMA DEL ESTADO Y DE LA ADMINISTRACIÓN PÚBLICA, 12., 2007, Santo Domingo, Rep. Dominicana. Anais... Santo Domingo, Rep. Dominicana: CLAD, 2007.

ANNUNZIATA, R. Los mecanismos participativos como puesta en escena de la proximidad. La experiencia del municipio de Morón. In: NARDACCHIONE, G. (Comp.). Todos juntos: dispositivos de participación de los gobiernos locales en la Argentina creciente. Buenos Aires: UNGS-Prometeo, 2011.

ARCIDIÁCONO, P.; KALPSCHTREJ, K.; BERMÚDEZ, Á.

¿Transferencias de ingresos, cooperativismo o trabajo asalariado? El Programa Argentina Trabaja. Trabajo y Sociedad, Santiago del Estero, Argentina, n. 22, jun. 2014.

ARGENTINA. Ministerio de Desarrollo Social de La Nación. Informe de Ejecución del Programa Argentina Trabaja: principales resultados al primer semestre de 2011. Buenos Aires, 2011.

BLANCO, I.; GOMÁ, R. Del gobierno a la gobernanza.

Oportunidades y retos de un nuevo paradigma": Polítika: Revista de Ciencias Sociales, Abendua, n. 2, p. 11-27, 2006. 
; SUBIRATS, J. ¿Existen territorios socialmente excluyentes? Contra lo inexorable. In: BLANCO, I.; FLEURY, S.; SUBIRATS, J. (Coords.). Innovaciones locales ante inseguridades globales: los casos de Brasil y España. Barcelona: Fundación CIDOB, 2008.

CABANNES, Y. Presupuesto participativo y finanzas locales. $2^{\mathrm{a}}$ versión ampl. Porto Alegre: Programa de Gestión Urbana - UN/ HABITAT, 2005.

CABRERO MENDOZA, E. Acción pública y desarrollo local. México: Fondo de Cultura Económica, 2005.

CANTO CHAC, M. Gobernanza y participación ciudadana en las políticas públicas frente al reto del desarrollo. Revista Política y Cultura, México, n. 30, 2008.

CAO, H. (Coord.). Introducción a la Administración Pública argentina. Buenos Aires: Editorial Biblos, 2007.

CARMONA, R. El debate sobre nuevos estilos de gobierno en ciudades argentinas. Buenos Aires: Ediciones CICCUS-UNGS, 2012a.

La dinámica sociopolítica y territorial en la implementación de intervenciones públicas en el ámbito local. Algunas consideraciones sobre tres distritos de la Región Metropolitana de Buenos Aires. Revista Territorios, Bogotá, v. 26, p. 107-128, 2012 b.

; COUTO, B. El Presupuesto Participativo como política innovadora de articulación entre el Estado y la sociedad. Un análisis de sus alcances en términos político-institucionales y de participación ciudadana para el contexto argentino, In: INTERNATIONAL CONFERENCE ON PUBLIC POLICY, Milan, 2015. Anais... Milan, 2015.

CRAVACUORE, D; ILARI, S.; VILLAR, A. La articulación de la gestión municipal. Actores y políticas. Buenos Aires: Universidad Nacional de Quilmes, 2004.

CUNILL GRAU, N. (1991) Participación ciudadana: dilemas y perspectivas de los Estados Latinoamericanos. Caracas: CLAD, 1991.

FORNI, P.; CASTRONUOVO, L. Ni piqueteros ni punteros: organizaciones populares durante el kirchnerismo. La Plata: EDULP, 2014.

GUIMÉNEZ, S.; HOPP, M. Programa Ingreso Social con Trabajo "Argentina Trabaja": una mirada reflexiva desde el corazón de su implementación. Buenos Aires: UBA, 2012. Trabajo realizado en el 
marco del Grupo de Estudios sobre Políticas Sociales y Condiciones de Trabajo.

JORDANA, J. Las relaciones intergubernamentales en la descentralización de las políticas sociales. Barcelona: Fundación CIDOB, 2003.

LECHNER, N. Three Forms of Social Coordination. CEPAL Review, Santiago, Chile, n. 61, apr. 1997.

LÓPEZ ACCOTTO, A. et al. La provincia de Buenos Aires y sus municipios: los laberintos de una distribución anacrónica de recursos. Buenos Aires: Editorial UNGS, 2014.

LOZANO GONZÁLEZ, C. Institucionalismo, gobernanza y sociedad civil. Revista IAPEM, México, n. 72, p. 37-62, ene./abr. 2009.

LOZANO, J. P. De la movilización a la institucionalización: la experiencia de organizaciones sociales de matriz nacional popular en el gobierno de la Provincia de Buenos Aires durante el periodo 2002-2010. In: TEJERINA, B.; PERUGORRÍA, I. (Ed.). Global Movements National Grievances: movilizing for "real democracy" and social justice. Espanha: Universidad del País Vasco, 2012. p. 356376.

MANIN, B. Los principios del gobierno representativo. Madrid: Alianza, 1998.

MARTÍNEZ, C.; ARENA, E. Experiencias y buenas prácticas en presupuesto participativo. Buenos Aires: UNICEF Argentina, 2013.

NATALUCCI, A. Aportes para la discusión sobre la autonomía o heteronomía de las organizaciones sociales, Movimientos Barrios de Pie. Lavboratorio: Revista de Estudios sobre Cambio Estructural y Desigualdad Social, Buenos Aires, ano 10, n. 23, p. 90-108, 2010. Disponivel em: $<$ http://www.lavboratorio.sociales.uba.ar/textos/ lavbo23_5.pdf>. Acesso em:

SÁNCHEZ, F. Orçamento Participativo: teoria e práctica. Porto Alegre: Cortez, 2002.

SUÁREZ, A. L.; GROISMAN, F. Segregación residencial y logros educativos en Argentina. In: R. KAZTMAN, R.; QUEIROZ RIBEIRO, L. (Org.). A cidade contra a escola: segregação urbana e desigualdades educacionais em grandes cidades da América Latina. Río de Janeiro: Letra Capital Editora, 2007. p. 33-58.

SVAMPA, M. Cambio de época: movimientos sociales y poder político. Buenos Aires: Siglo Veintiuno Editores, 2009. 
POLÍTICAS DE PARTICIPACIÓN CIUDADANA EN AMBITOS LOCALES DE ARGENTINA: alcances en términos políticos y de gestión pública

\section{NOTAS}

1 Barrios de Pie, Federación de Tierra y Vivienda, Central de Trabajadores Argentinos, Libres del Sur, y miembros de la UNGS.

2 El Presupuesto asignado dentro del MDS al AT ocupa el segundo mayor porcentaje del gasto total en 2010 y 2011 ( $21 \%$ y 13\% respectivamente del presupuesto ejecutado), ubicándose debajo del gasto por Pensiones no contributivas que representa el $57 \%$ u $66 \%$ en esos mismos años. (ARCIDIÁCONO; KALPSCHTREJ; BERMÚDEZ, 2014). 
